\title{
Der Begriff Heterogenität in empirischen Studien
}

\section{Sandra Zulliger und Sabine Tanner}

Welche Themen und Heterogenitätskategorien werden erforscht, wenn im Titel einer empirischen Studie der Begriff „Heterogenität" verwendet wird? Anhand einer systematischen Datenbankrecherche und Inhaltsanalyse von empirischen Studien wird dieser Frage nachgegangen. 21 Studien entsprachen den definierten Kriterien und lassen sich in die folgenden drei Themenschwerpunkte aufteilen: 1. Die Heterogenität der Schülerschaft in Bezug auf unterschiedliche Heterogenitätskategorien und Analyseebenen. Die Leistungsheterogenität ist in diesem Zusammenhang die am häufigsten untersuchte Heterogenitätskategorie. 2. Die Anwendung, Evaluierung und Entwicklung von integrativen Unterrichtsmethoden. 3. Die subjektiven Theorien der Lehrpersonen zur Heterogenität ihrer Schülerschaft.

\section{Einle itung}

Der Begriff Heterogenität ist in den letzten Jahren zu einem wichtigen Begriff der deutschsprachigen Erziehungswissenschaft avanciert. International vergleichende Schulleistungsstudien wie bspw. PISA, TIMSS oder IGLU tragen dazu bei, dass Heterogenität als Sammelbegriff eines mit vielerlei Herausforderungen behafteten Themenfeldes in einer zunehmend breiteren Öffentlichkeit diskutiert wird (vgl. z.B. Wenning, 2007).

Wie sich in den folgenden Ausführungen zur Begriffsbestimmung zeigen wird, handelt es sich bei Heterogenität um einen sehr vielschichtigen Begriff, der viele Unklarheiten offen lässt. Zur Begriffsbestimmung werden u.a. Listendefinitionen ausgeführt, welche die heterogenitätsstiftenden Schülermerkmale nennen (z.B. Wischer, 2009). Schülerinnen und Schüler können sich diesem Verständnis zufolge theoretisch in unendlich vielen verschiedenen Merkmalen unterscheiden. Im Schulkontext wird der Fokus dabei häufig auf Merkmale gerichtet, die in Zusammenhang mit dem Lern- und Leistungsverhalten resp. dem resultierenden Bildungserfolg von Interesse sind (Stöger \& Ziegler, 2012). Eigenschaften des Heterogenitätsbegriffs fassen Lang, Grittner, Rehle und 
Hartinger (2010) in vier Punkten zusammen:

- Relativität: Heterogen ist keine absolute Eigenschaft. Was als heterogen bezeichnet wird, ist das Ergebnis eines Vergleichs eines bestimmten Kriteriums zu einem bestimmten Massstab.

- Partialität: Mit Heterogenität werden nur zeitlich begrenzte Zustände beschrieben, da sich die Unterschiede wieder vergrössern oder verringern können. Zudem gilt die Bezeichnung nur partiell und kann nicht verwendet werden, um Schülerinnen und Schüler oder eine Gruppe von Schülern als Ganzes oder deren zukünftige Entwicklung zu beschreiben.

- Konstruiertheit: Erst durch das Anlegen eines bestimmten Massstabs kann Heterogenität bzw. Homogenität bestimmt werden. Es handelt sich somit um ein Konstrukt, das vom jeweiligen Massstab abhängig ist.

- Wertneutralität: Die Konstruktion von Heterogenität bzw. Homogenität ist nicht grundsätzlich gut oder schlecht. Sie erfolgt jedoch immer aus einem bestimmten Interesse und in bestimmten Zusammenhängen. Lernrelevante Unterschiede der Schülerinnen und Schüler werden z.B. häufig normativ interpretiert.

Zur Bezeichnung der Unterschiedlichkeit von Personen findet neben dem Begriff der Heterogenität häufig der Begriff Diversity / Diversität Verwendung. Die beiden Begriffe erzeugen nach Krüger-Potratz (2011) je unterschiedliche Vorstellungen. Diversity / Diversität ist überwiegend im ökonomischen Bereich im Sinne von "Diversity Management» in Gebrauch. Diversity Management bezeichnet die Berücksichtigung der Vielfalt und der Verschiedenheit des Personals zur Effizienzsteigerung. Im Gegensatz zu Diversität / Diversity ist der Begriff Heterogenität binär codiert. Der Gegenbegriff Homogenität ist eher positiv besetzt und bezeichnet Einheitlichkeit. Der hauptsächlich im schulischen Kontext verwendete Begriff Heterogenität ist hingegen vielfach mit Schwierigkeiten assoziiert. Diesen Schwierigkeiten gilt es im Schulalltag entgegenzuwirken (Krüger-Potratz, 2011).

Das System Schule ist in seiner Grundstruktur darum bemüht, Homogenität herzustellen. In diesem Sinne werden Schülerinnen und Schüler nach Alter und Leistung gruppiert (Leiprecht \& Lutz, 2006). Es ist unbestritten, dass die schulische Leistung das zentrale Ordnungskriterium des heutigen Schulsystems repräsentiert (Lindemann, Schwanenberg \& Bos, 2011). Angesichts der Aktualität und Brisanz von Schulleistungsunterschieden wird angenommen, dass die Leistungsheterogenität in empirischen Studien einen zentralen Untersuchungsgegenstand darstellt. Eine in jüngster Zeit aufgekommene Forderung an die Forschung ist dabei, nach Möglichkeit mehrere Heterogenitätsdimensionen miteinander zu betrachten, um z.B. die Ursachen von Schulerfolg oder -misserfolg aufschlussreicher zu ergründen (Prengel, 2007).

Die aufgezeigten Begriffsannäherungen lassen im Zusammenhang mit der Verwendung des Begriffs Heterogenität in empirischen Studien vielerlei Fragen 
aufkommen. Welche Schülermerkmale werden herangezogen, um Heterogenität festzustellen? Welche Massstäbe werden angelegt? Für diesen Beitrag ist die Forschungsfrage wegleitend, was genau erforscht wird, wenn im Titel eines Beitrags von Heterogenität die Rede ist.

Folgende Fragestellungen werden ausformuliert:

1. In welchem thematischen Kontext wird der Begriff,Heterogenität' in empirischen Studien aus dem Schulumfeld eingebracht?

2. Welche Heterogenitätskategorien stehen im Mittelpunkt des Forschungsinteresses?

Mit der Analyse dieser Fragestellungen sollen die übergeordneten Themengebiete der verschiedenen Beiträge und die verwendeten Heterogenitätskategorien aufgezeichnet werden. Die Studie leistet dabei keinen Beitrag zur genaueren Begriffsbestimmung, sondern zeigt lediglich die Bandbreite der inhaltlichen Anwendungen auf. Im Folgenden wird das methodische Vorgehen erläutert. Im Ergebnisteil und der Zusammenschau werden die zentralen Erscheinungsformen herausgearbeitet, die den Begriff Heterogenität im schulischen Kontext kennzeichnen. Das Forschungsvorgehen bezweckt insofern das Rekonstruieren und Aufschlüsseln von Realität (Diaz-Bone, 2006). Dies ist für den pädagogischen Heterogenitätsdiskurs der Gegenwart wünschenswert, da der Umgang mit Heterogenität als grosse zukünftige Herausforderung der Schule gilt (Wischer, 2007).

\section{Methodisches Vorgehen}

Die Zusammenstellung der wissenschaftlichen Veröffentlichungen folgt dem Interesse, die empirische Sättigung des Begriffs Heterogenität in Forschungsstudien zu erfassen. Bestandteil und Grundlage der Suche ist der Begriff Heterogenität, verschränkt mit der Suche nach empirischen Studien. Konkret wird nach Einzelbeiträgen gesucht, die sich mit Heterogenität befassen und diesen Begriff im (Unter-)Titel aufführen. Beim (Unter-)Titel kann davon ausgegangen werden, dass dieser von der Autorenschaft gewählt wurde und somit bewusst ein Einbringen in den Heterogenitätsdiskurs angestrebt wird. Im Gegensatz dazu verfügt der Heterogenitätsbegriff im Abstract oder in der Verschlagwortung des Beitrags über eine geringere Priorität, da beide nicht zwingend auf die Autorenschaft zurückgehen und somit nicht mit einer entsprechenden Absicht in Zusammenhang stehen müssen.

Im Rahmen dieser Untersuchung wird eine systematische Forschungsreview angestrebt. Systematische Reviews zeichnen sich gemäss Petticrex und Roberts (2006, zitiert nach Steinke \& Badura, 2011) durch folgende Merkmale aus: 
- präzise, operationalisierte Fragestellung

- definierte Ein- und Ausschlusskriterien, die zu Beginn der Recherche festgelegt werden

- eine systematische und umfassende Recherche, die sicherstellt, dass alle relevanten Studien zu der untersuchten Fragestellung berücksichtigt werden

- strukturierte und transparente Darstellung der Ergebnisse und insbesondere der Vorgehensweise

Um die Fragestellung beantworten zu können, anerbietet sich eine Eingrenzung der Recherche auf den deutschsprachigen Raum. Somit werden Ergebnisse hervorgebracht, die für den Gebrauch des Heterogenitätsbegriffs im schweizerischen, im deutschen und im österreichischen Forschungskontext aussagekräftig sind.

Als Datengrundlage wird die deutschsprachige FIS Bildung Literaturdatenbank ausgewählt. Sie repräsentiert die für den deutschsprachigen Raum wichtigste und umfangreichste Metadatenbank. In die Datenbank werden Artikel aus ca. 430 Fachzeitschriften, Monografien, Beiträge aus Sammelwerken und elektronische Dokumente aus Datenbankbeständen von ca. 30 Dokumentationseinrichtungen in Deutschland, der Schweiz und Österreich einbezogen (Fachportal Pädagogik, 2012).

Die Recherchestrategie charakterisiert sich dadurch, dass Beiträge ausgewählt werden, die im Titel oder Untertitel den Begriff ,Heterogenität' mitführen und zudem eine empirische Studie darstellen, die von der Urheberschaft selbst publiziert wurde. Um sicherzugehen, dass es sich beim ausgewählten Dokument auch wirklich um eine empirische Studie handelt, wurden aus dem Schlagwortregister alle ähnlichen oder abgewandelten Begriffe zu „Empirie', ,Untersuchung', ,Forschen' und ,Studie' einbezogen und mit der Suche nach dem Begriff ,Heterogenität' im Titel und im Untertitel verknüpft. Thematisch berücksichtigt wurden Beiträge aus dem Schulumfeld, in welchen sich der Begriff Heterogenität auf die Schülerinnen und Schüler bezieht. Bezüglich der Schulstufen wurden Studien der Primarstufe, der Sekundarstufe I und der Sekundarstufe II (Gymnasium, Berufsbildung) aufgenommen. Empirische Studien zur Heterogenität von Studierenden und Erwachsenen werden nicht einbezogen. Themen, die Kinder und Jugendliche betreffen, jedoch nicht klar erkennbar mit dem schulischen Kontext in Verbindung stehen (z.B. Mädchenarbeit, Medienkonsum) werden ausgeschlossen.

Nebst den inhaltlichen Merkmalen zeichnet sich die durchgeführte Recherche durch folgende Formalitäten aus:

- Einbezug aller Dokumentationstypen: Monografien, Sammelwerksbeiträge und Zeitschriftenaufsätze. Das ursprüngliche Vorhaben, nur Zeitschriftenartikel aufzunehmen, die ein Peer-Review-Verfahren durchlaufen haben, wurde u.a. aus dem Grund wieder fallen gelassen, als pädagogische Forschungsbeiträge nicht ausschliesslich in Zeitschriften publiziert werden, sondern $\mathrm{zu}$ 
einem grossen Teil in Sammelwerken Aufnahme finden.

- Ausgeschlossen werden publizierte Vorträge und Publikationen aus nicht von einem Fachpublikum rezipierten Zeitschriften wie z.B. dem ,Schulblatt Aargau'.

- Bei mehreren Beiträgen aus dem gleichen Forschungsprojekt wurde die jeweils aktuellste Publikation in die Analyse aufgenommen.

- Es fand eine Eingrenzung auf die Sprache Deutsch statt.

- Zeitlich wurde keine Beschränkung vorgenommen.

Insgesamt ergab die Recherche 162 Treffer (Stichtag der Suche: 15. Juni 2012). Sämtliche Beiträge wurden auf alle genannten Selektionskriterien hin überprüft. Viele Beiträge wurden ausgegrenzt, da sie nicht von der Urheberschaft der Studie selber publiziert wurden ${ }^{1}$. Übrig blieben 21 Beiträge, die allen Kriterien entsprachen und somit als Datengrundlage für die vorzunehmende Analyse herangezogen wurden. Die ausgewählten Studien werden dabei keiner Qualitätsprüfung unterzogen, sondern lediglich hinsichtlich der interessierenden Inhaltsdimensionen analysiert.

\section{Ergebnisse}

Die 21 Beiträge wurden zwischen 2001 und 2011 publiziert. Es handelt sich um 3 Dissertationen aus den Jahren 2009, 2010 und 2011, 11 Sammelwerksbeiträge, von denen 2 im gleichen Werk erschienen sind, und um 7 Zeitschriftenaufsätze. 2 Beiträge stammen aus Österreich, 6 aus der (Deutsch-)Schweiz und 13 aus Deutschland. Auffallend ist hier, dass eine relativ geringe Zahl empirischer Studien den Begriff Heterogenität im Titel bringt. Es fehlen z.B., ausser der IGLU-Studie, die wichtigen internationalen Studien wie die PISA-Studie oder Studien, die verschiedene Aspekte von Heterogenität ausdrücklich thematisieren (z.B. EDK-ost 4bis8). Dennoch ist die Datenbasis breit genug, um in einem explorativen Verfahren Aussagen zur Verwendung des Begriffs Heterogenität in empirischen Studien zu generieren.

Allgemein fällt die unterschiedlich häufige Verwendung des Begriffs Heterogenität innerhalb der Beiträge auf. Neben der Verwendung im Titel reicht die Spannweite von einer zweimaligen Nennung des Begriffs im Text (z.B. Allemann-Ghionda, Auernheimer \& Grabbe, 2006) bis zu einem Versuch der Begriffsbestimmung (Grunder, 2009) und näheren Erläuterungen des Begriffs (Schründer-Lenzen, 2008). Der (Unter-)Titel des Texts gibt dabei teilweise schon Auskunft über die behandelten Heterogenitätskategorien - z.B. «Lernentwicklung in leistungshomogenen und -heterogenen Schulklassen» (Bos \& Scharenberg, 2010). Bei anderen Beiträgen sind die verwendeten Heterogenitätskategorien aus dem Titel nicht ersichtlich wie z.B. in «Homogene oder heterogene Schulen oder Klassen?»(Wirszing \& Weichert, 2008). 
Thematisch eingeführt werden die Beiträge häufig, wie dies Budde (2012) auch bereits angemerkt hat, mit dem Hinweis auf die PISA-Studien (z.B. Schneider, 2009), das stark selektive Schulsystem im deutschsprachigen Raum (z.B. Schratz, Schwarz \& Westfall-Greiter, 2011) oder mit dem Hinweis auf die grosse und stetig steigende Heterogenität der Schülerschaft (z.B. Schenz, 2011).

Aus den einzelnen Beiträgen geht die grobe Kategorisierung in die folgenden Themengebiete hervor:

1. Schülerinnen und Schüler: Heterogenität in der Schule.

2. Unterricht: Forschungsstudien (Interventionsstudien, Evaluationen) zu didaktischen Methoden/pädagogischen Handlungsweisen.

3. Lehrpersonen: Unterrichten in heterogenen Klassen; Einstellungen der Lehrpersonen.

Die drei Themengebiete werden im Folgenden mit Hilfe der analysierten Beiträge genauer vorgestellt und konkretisiert.

\section{Schülerinnen und Schüler}

Unterschiede zwischen den Schülerinnen und Schülern an der Schule stehen hier im Vordergrund. Die acht Beiträge zu diesem Themengebiet lassen sich verschiedenen Ebenen zuordnen. Eine Zuordnung kann hinsichtlich der jeweils obersten Analyse- und Vergleichsebene unternommen werden, nämlich auf Ebene des Individuums, der Schulklasse, der Schule oder auf Ebene eines Ländervergleichs.

Individuum: Zwei Studien untersuchen die Lernprozesse einzelner Individuen. Die Mikroebene von Lernprozessen und die interindividuellen Unterschiede stehen hier im Vordergrund. Die qualitativ ausgerichtete Studie von Schratz et al. (2011) untersucht die Bedeutungen von Lernerfahrungen für die Lernenden. Schneider (2009) ermittelt mit einer Längsschnittstudie die literale Resilienz von Kindern mit ungünstigen Lernbedingungen. Die Studie kontrolliert bei den Schülerinnen und Schülern die Merkmale Geschlecht, Sozialstatus, kognitive Grundfähigkeiten, Migrationshintergrund und untersucht im Besonderen deren Leseleistung. Der Begriff Heterogenität wird in beiden Studien im Titel erwähnt, ohne dass im Text darauf Bezug genommen wird. Schratz et al. (2011) zeigen am Beispiel eines Mädchens mit Migrationshintergrund die Erfassung personaler Bildungsprozesse auf. Insgesamt wurden 48 Schülerinnen und Schüler untersucht, von denen angenommen wird, dass sie einer heterogenen Gruppe entstammen, da es «aufgrund der demographischen, wirtschaftlichen und gesellschaftlichen Entwicklungen zu einer starken Durchmischung der Schülerinnen und Schüler an allen Schularten» (Schratz et al., 2011, S. 26) kommt. Die Heterogenität der Schülerschaft wird hier implizit vorausgesetzt, ohne ein genaueres Augenmerk auf deren Ausprägungen zu werfen. 
Schulklasse: Die Schulklasse ist eine durch das Schulsystem gegebene Gruppierung von Schülerinnen und Schülern, die gemeinsam beschult werden. Dementsprechend häufig wird die Schulklasse als Bezugssystem und Referenzpunkt für Vergleiche herangezogen. Die Schülerinnen und Schüler werden innerhalb ihrer Schulklassen untereinander verglichen oder es finden Vergleiche zwischen den Schulklassen statt.

Die Altersheterogenität ist Thema der einen Studie, welche die Zusammensetzung von in Berufsschulen ,natürlich' vorkommenden altersheterogenen Lerngruppen untersucht (Reinhardt \& Rosen, 2004). Der Begriff Altersheterogenität wird hier in Abgrenzung zum Begriff Altershomogenität verwendet.

Eine einzelne Studie aus der Integrationsforschung befasst sich mit der Ausgrenzung von Schülerinnen und Schülern mit Behinderungsmerkmalen und dem Zusammenhang zu verschiedenen Schülermerkmalen (Huber, 2009). Die Untersuchung findet in Klassen statt, in welche ein Kind mit besonderem Förderbedarf integriert ist. In diesen Klassen werden alle Kinder gemeinsam unterrichtet. Insgesamt 11 Schülermerkmale (Intelligenz, Schulleistung, Konzentration, Motivation, Selbstständigkeit, Sozialkompetenz, Alter, sozialer Rückzug, Aggressivität, Belastbarkeit und Selbstreflexion) werden erfasst und normiert. Die Varianz dieser Merkmale bildet die Lerngruppenheterogenität in den einzelnen Klassen ab und wird mit anderen Schulklassen (insgesamt 30 Schulklassen) verglichen. Auf den Begriff Heterogenität wird explizit Bezug genommen. «Heterogenität ist ein abstrakter Begriff, der hier als möglichst vielfältige und häufige Abweichung von einer Gruppennorm betrachtet wird» (Huber, 2009, S. 181). Der Begriff der Norm wird hier im Sinne der häufigsten Ausprägung verwendet.

Eine weitere Untersuchung, die einzelne Schulklassen miteinander vergleicht, ist die KILIA-Studie von Martschinke und Kammermeyer (2003). Fragestellungen sind: Wie sehen Klassen im Anfangsunterricht bezüglich der leistungsund persönlichkeitsspezifischen Lernvoraussetzungen Leistung (phonologisches Bewusstsein, Selbstkonzept im Schriftspracherwerb, Aspekte der Persönlichkeit wie z.B. leistungsbezogene Angst, Selbstkonzept usw.) und Kontextvariablen wie z.B. der Klassengrösse und der Elternunterstützung aus? Welche Unterrichtsmassnahmen (Differenzierung, Freiheitsspielräume) werden von den Lehrkräften ergriffen und wie hängen diese mit der Heterogenität der vormals aufgezeigten Merkmale der jeweiligen Klasse zusammen? Die Studie gilt es hervorzuheben, da sie die Ebene der Schulklasse mit derjenigen der Lehrperson verknüpft und aufzuzeigen versucht, wie die Lehrperson auf die im Anfangsunterricht vorgefundenen Bedingungen, d.h. die Heterogenität der Schülerschaft, reagiert. Die Komplexität des Unterrichts wird mit verschiedenen Heterogenitätskategorien in Zusammenhang gebracht. Eine weitere Studie, die diese beiden Ebenen miteinander verknüpft, ist die explorative Studie von Eckhart (2002), welche die Unterrichtsformen fokussiert (genauer erläutert und eingeordnet wird die Studie unter dem Thema Unterricht, s. unten). 
Die weiteren Beiträge auf der Ebene Schulklasse behandeln die Klassenunterschiede zwischen verschiedenen Schulformen. Anders als in den vorangehenden Beiträgen wird nicht mit Absolutwerten gerechnet, sondern mit den Unterschieden zwischen vermeintlich homogeneren und heterogeneren Schulklassen. Im Forschungsbeitrag von Amberg und Posch (2004) werden die nach dem herkömmlichen Schulmodell gebildeten altershomogenen Schulklassen bezüglich ihrer Leistung und dem Sozialverhalten mit altersgemischen Modellen wie der flexiblen Schuleingangsphase und den Mehrstufenklassen verglichen.

Schule/Schulform: Wirszing und Weichert (2008) vergleichen zwei Schulen miteinander bezüglich der Persönlichkeitsentwicklung ihrer Abgängerinnen und Abgänger. Die «sehr heterogene» Schule unterscheidet sich von der «normal heterogen und homogenisierenden» (Wirszing \& Weichert, 2008, S. 11) Schule durch ein multikulturelles Einzugsgebiet und «baut zudem auf ein Schulkonzept, welches darüber hinaus Heterogenität über das natürliche Mass hinaus ,herstellt'» (ebd., S. 11), dies mit jahrgangsübergreifenden Integrationsklassen und gezieltem sozialen Bezugsgruppenwechsel. Die homogene Schule weist die «klassische Heterogenität einer Jahrgangsschule» (ebd., S. 11) auf.

Ländervergleich: Aus einer soziologischen Perspektive wird im letzten Beitrag die Verschiedenheit zwischen den Schülerinnen und Schülern betrachtet. Im Beitrag zur IGLU-Studie von Schwippert, Bos und Lankes (2004) wird anhand von Schüler- und Elternbefragungen der Zusammenhang zwischen den Leistungen in Lesen, Mathematik und den Naturwissenschaften mit der Sozialschicht, dem Migrationshintergrund und dem Geschlecht ermittelt. Die Ergebnisse der Studie können zwar auch für den Vergleich von einzelnen Schulklassen verwendet werden, hier steht jedoch der Vergleich mit anderen Ländern im Vordergrund.

\section{Unterricht}

Die folgenden sieben Beiträge fokussieren den Unterricht und untersuchen die Anwendung von einzelnen Unterrichtsmethoden, welche besonders für eine heterogene Schülerschaft gedacht sind. Anhand von Interventionsstudien und/ oder Evaluationen werden didaktische Methoden und pädagogische Handlungsweisen überprüft.

Kontext dieser Untersuchungen ist die Kritik an der äusseren Differenzierung und der damit verbundenen Aussonderung von Kindern, die besonderer Förderung bedürfen (siehe z.B. Eckhart, 2002). In diesem Zusammenhang werden Unterrichtsmethoden vorgestellt, die einen integrativen Unterricht erlauben und insbesondere auch Schulleistungsschwachen und/oder Kindern mit Migrationshintergrund zugutekommen sollen.

Eine Studie, die das Unterrichtsgeschehen aufnimmt, ist diejenige von Hugener, Krammer und Pauli (2008). Anhand von gefilmten Mathematiklektionen wird das Differenzierungsrepertoire von einzelnen Lehrpersonen erfasst.

Eckhart (2002) beschreibt die Ergebnisse einer explorativen Studie und 
inwieweit sich integrative didaktisch-methodische Vorgehensweisen auf den Lernerfolg sowie auf die soziale Integration von in- und ausländischen Kindern mit Schulleistungsschwächen auswirken. Hierzu wurden bei den Schülerinnen und Schülern die soziale Integration und die Intelligenz erfasst. Bei den Lehrpersonen wurden Angaben zur Verwirklichung von integrativen Unterrichtsformen (innere Differenzierung, zieldifferenziertes Lernen, fächerübergreifendes Lernen, offene Lernformen) eingeholt. Wie bereits im vorangehenden Kapitel erwähnt, wird hier die Ebene der Schülerinnen und Schüler mit derjenigen der Lehrpersonen verknüpft.

Drei Interventionsstudien mit anschliessender Evaluation untersuchen erstens kooperative Lernformen hinsichtlich Leistung und ethischen Hintergrunds von heterogenen Lerngruppen und deren integrationsfördernde Wirkung (Lanphen, 2001), zweitens eine für Schülerinnen und Schüler mit ungünstigen Lernvoraussetzungen $^{2}$ gestaltete adaptive Unterrichtseinheit (kognitive Strukturierung, Peer Learning und formatives Assessment) (Warwas \& Kunter, 2011) und drittens Fördermassnahmen (FörMig), welche insbesondere für Schülerinnen und Schüler mit Migrationshintergrund gedacht sind (Schründer-Lenzen, 2008).

Eine weitere Studie ist diejenige von Wischer (2001), welche die Absolventinnen und Absolventen der Laborschule Bielefeld analysiert. An der Laborschule werden leistungsheterogene Gruppen bis zum Abschluss des 9. Schuljahres unterrichtet. Es wird untersucht, wie erfolgreich die innere Differenzierung des Unterrichts aus Sicht der Schülerinnen und Schüler durchgeführt wird, und ob, insbesondere bei den Leistungsschwächeren, ein Effekt auf das Selbstkonzept und das Wohlbefinden nachweisbar ist.

Ein Dissertationsprojekt befasst sich mit der Herausarbeitung von Best-Practice für die Basisstufe. Mittels teilnehmender Beobachtung werden günstige pädagogische Handlungsweisen abgeleitet, welche insbesondere auch benachteiligten Kindern, d.h. Kindern, deren Erstsprache nicht Deutsch ist und/oder die aus einem bildungsfernen Milieu stammen, gerecht werden (Urech, 2010).

Auffallend an den zum Thema Unterricht eingeordneten Studien ist die Verwendung der Heterogenitätskategorien. Bei der Studie von Hugener et al. (2008), welche das Unterrichtsgeschehen bezüglich der Methoden der inneren Differenzierung thematisiert, werden Heterogenitätskategorien nicht explizit erwähnt. Bei den übrigen Studien zum Unterricht stehen die Merkmale Migrationshintergrund und Leistungsschwäche in Kombination mit einer für alle Schülerinnen und Schüler gedachten Unterrichtsmethode im Fokus des Interesses. Die letztgenannte Untersuchung von Urech (2010) ist als Ausnahme zu bezeichnen, da es um die Entwicklung von pädagogischem Handlungswissen (Best-Practice) für eine neu organisierte, ,heterogenitätsfördernde' Schulform geht. Charakteristiken, die einen gelingenden Unterricht in der Basisstufe ausmachen, werden herausgearbeitet. 


\section{Lehrpersonen}

Bei Beiträgen, die dieser Thematik zugeteilt wurden, liegt der Fokus auf der Lehrperson und deren Umgang mit Heterogenität in Schule und Unterricht. Leitend ist jeweils die Frage, wie Lehrpersonen mit Heterogenität oder der schulischen Selektionsfunktion umgehen. Die Heterogenitätskategorien werden hierbei nicht genauer fokussiert, sondern es wird davon ausgegangen, dass die Schülerschaft per se heterogen ist. Die subjektiven Theorien der Lehrpersonen stehen im Vordergrund. In sämtlichen Beiträgen wurden Lehrpersoneninterviews als Erhebungsmethode eingesetzt.

Den Lehrpersonen obliegt die Aufgabe der Förderung wie auch der Auslese. Der Aspekt der Auslese steht in einer soziologisch und einer pädagogisch ausgerichteten Studie im Vordergrund. In der soziologisch ausgerichteten Studie werden vor dem Hintergrund der Reproduktion von Ungleichheit nach Bourdieu Lehrpersonen nach dem Spannungsfeld zwischen Förderung und Selektion sowie ihrem Heterogenitätsverständnis befragt (Streckeisen, Hänzi \& Hungerbühler, 2009). In der pädagogisch ausgerichteten Studie von Allemann-Ghionda et al. (2006) wird mit explorativen Interviews die diagnostische Kompetenz von Lehrpersonen im Hinblick auf Kinder mit Migrationshintergrund untersucht.

Das professionelle Verständnis der Lehrpersonen im Umgang mit einzelnen Heterogenitätskategorien wird in drei Studien erfasst. Dies mit Blick auf (hoch) begabte Kinder und Jugendliche bei Gymnasiallehrpersonen (Schenz, 2011), in Bezug auf kulturelle Heterogenität (Edelmann, 2006) und in einem Dissertationsprojekt zur vergleichenden Betrachtung von deutschen und französischen Lehrpersonen zu ihrem Umgang mit soziokultureller Vielfalt (Sievers, 2009).

\section{Zusammenschau}

Mit den angewendeten Methoden konnten empirische Studien identifiziert und analysiert werden, welche sich explizit mit Heterogenität befassen und sich bewusst in den Heterogenitätsdiskurs einbringen. Die Themenbereiche und aufgegriffenen Heterogenitätskategorien wurden aufgeschlüsselt und werden im Folgenden zusammenfassend dargestellt. Eine wichtige Erkenntnis ist, dass je nach Themenbereich andere Heterogenitätskategorien aufgegriffen werden. Allerdings ist die Ausdifferenzierung in verschiedene Heterogenitätskategorien nicht in allen Themenbereichen von gleicher Relevanz.

Relativierend wird angemerkt, dass eine (weit grösser gefasste) Analyse unter Einbezug aller empirischen Studien, welche sich mit Heterogenität(skategorien) befassen, ev. zu anderen Ergebnissen gekommen wäre. Die Datenbasis von 21 Studien erlaubt im Sinne einer explorativen Auswertung, Aussagen zur thematischen Breite und den verwendeten Kategorien zu machen. Des Weiteren sind die konkreten Ergebnisse der untersuchten Studien und insbesondere das Ensemble der in den einzelnen Beiträgen erforschten Heterogenitätskategorien 
nicht Bestandteil des vorliegenden Beitrags, könnten jedoch Anlass für an diese Studie anschliessende Forschungsfragen sein.

In geraffter Form werden die ermittelten Themenbereiche der empirischen Studien wie folgt dargestellt:

\section{Tabelle 1: Themenbereiche der 21 untersuchten empirischen Studien}

\begin{tabular}{|l|l|l|}
\hline Schülerinnen und Schüler (9) & Unterricht (7) & Lehrpersonen (5) \\
\hline Analyseebene: & $\begin{array}{l}\text { Unterrichtsmethoden anwenden, } \\
\text { evaluieren, entwickeln (5) }\end{array}$ & $\begin{array}{l}\text { Subjektive Theorien der } \\
\text { Lehrpersonen zu Selek- } \\
\text { tionsfunktion; Umgang } \\
\text { Individuum (2) }\end{array}$ \\
Klasse (5) & Häufigkeit/Anwendung der inneren & Einstellung zu \\
Schule (1) & Differenzierung durch Lehrpersonen (2) & $\begin{array}{l}\text { (einzelnen) Heteroge- } \\
\text { nitätskategorien }\end{array}$ \\
\hline Ländervergleich (1) & & \\
\hline
\end{tabular}

In Klammern steht die Anzahl der Beiträge.

Die aus der systematischen Recherche hervorgegangenen Beiträge liessen sich eindeutig einem Themengebiet zuordnen (Ausnahmen: die Studien von Martschinke und Kammermeyer, 2003 und Eckhart, 2002).

Die in der Einleitung skizzierte, inhaltliche Vielschichtigkeit des Begriffs Heterogenität kommt in den ausgewählten empirischen Studien zum Tragen. Die Konstruiertheit und Relativität des Begriffs Heterogenität (vgl. Lang et al., 2010) zeigt sich insbesondere im Themenbereich Schülerinnen und Schüler, und zwar anhand der verschiedenen Analyseebenen, die vom Individuum bis hin zum Ländervergleich reichen. Die Unterschiede der Schülerinnen und Schüler resp. das Ausmass der Heterogenität wird in Relation zur jeweiligen Analyseebene beurteilt. Es sei an dieser Stelle an den Beitrag von Wirszing und Weichert (2008) erinnert, die eine «sehr heterogene» Schule mit einer «normal» heterogenen Schule vergleichen. In anderen Studien (z.B. Huber, 2009; Martschinke \& Kammermeyer, 2003) wurden in als ,normal ' heterogen geltenden Klassen/ Schulen Unterscheidungsmerkmale der einzelnen Schülerinnen und Schüler untersucht. Dabei wird auf den Umstand aufmerksam gemacht, dass scheinbar homogenisierte Gruppen auch heterogen sind. Bezüglich der untersuchten Heterogenitätskategorien bestätigte sich die in der theoretischen Einleitung aufgezeigte, zentrale Rolle der Leistungsheterogenität. Die Leistung bildete in den meisten Studien zusammen mit weiteren Aspekten, z.B. Persönlichkeit (Martschinke \& Kammermeyer, 2003) oder Sozialverhalten (Amberg \& Posch, 2004) die interessierende Zielgrösse oder erwies sich, wie in der Studie von Huber (2009), als zentrale Variable zur Aufklärung von Lerngruppenheterogenität. Ausnahmen bildeten die Studien von Reinhardt und Rosen (2004) zur Altersheterogenität, sowie Wirszing und Weichert (2008), welche die Persönlichkeitsentwicklung in Abhängigkeit verschiedener Schulformen analysierten.

Das in der Einleitung von Krüger-Potratz (2011) im Heterogenitätsbegriff erwähnte Mitschwingen von Schwierigkeiten und Konflikten lässt sich in den Beiträgen zum Themenbereich Unterricht und Lehrpersonen am deutlichsten 
erkennen. Bei Beiträgen, die diesen beiden Bereichen zugeordnet wurden, blieben Heterogenitätskategorien und die genaue Bestimmung, worin die Unterschiedlichkeiten der Schülerinnen und Schüler bestehen, im Hintergrund. Dass die Schülerschaft heterogen ist, wurde vorausgesetzt und dahingehend die Umsetzung von Unterricht sowie die Sichtweise der Lehrpersonen fokussiert. Insbesondere wurden von der jeweiligen Norm abweichende Heterogenitätsausprägungen wie Migrationshintergrund und die schulische Leistungsschwäche thematisiert sowie der Umgang mit entsprechenden Normabweichungen im Rahmen selektiver, aussondernder Schulsysteme kritisiert (z.B. Eckhart, 2002). Eine Ausnahme bildete die Studie von Schenz (2011), die Vorstellungen von Lehrpersonen über (Hoch-)Begabte thematisiert.

Hervorgehoben seien zum Schluss die KILIA-Studie von Matschinke und Kammermeyer (2003) und die explorative Studie von Eckhart (2002), welche die Ebene der Schülerinnen und Schüler und diejenige der Lehrpersonen miteinander verschränken. Beide Studien verbinden die Heterogenitätskategorien auf Ebene der Schülerinnen und Schüler mit der Ebene des Unterrichts und der Lehrperson. Unterschiedliche Heterogenitätskategorien werden dadurch kombiniert und zudem mit der Komplexität des Unterrichts in Zusammenhang gebracht. Studien mit entsprechend kombinierten Analyseebenen sind gegenüber Studien, die einzelne Ebenen isoliert untersuchen, aufgrund der dargestellten Ergebnisse klar in der Minderheit, jedoch für zukünftige Forschungsprojekte erwünscht.

\section{Anmerkungen}

1 Bspw. wird eine Publikation, die einen Überblick über verschiedene Studien leistet (und den Begriff Heterogenität im Titel führt), in der Analyse nicht berücksichtigt.

2 Der Beitrag ist sehr kurz gehalten, woraus keine Angaben zu einer genaueren Bestimmung dieser ungünstigen Lernvoraussetzungen entnommen werden können.

\section{Literatur}

Allemann-Ghionda, C., Auernheimer, G. \& Grabbe, H. (2006). Beobachtung und Beurteilung in soziokulturell und sprachlich heterogenen Klassen. Die Kompetenzen der Lehrpersonen. In C. Allemann-Ghionda (Hrsg.), Kompetenzen und Kompetenzentwicklung von Lehrerinnen und Lehrern. (Zeitschrift für Pädagogik, Beiheft 51, S. 250-266). Weinheim: Beltz.

Amberg, I. \& Posch, V. (2004). Schulleistungen und Sozialverhalten in altersheterogenen und altershomogenen Lerngruppen auf der Grundstufe. Eine empirische Untersuchung auf der 2. Schulstufe an Wiener Volksschulen. Erziehung \& Unterricht, 154 (1-2), 88-114.

Bos, W. \& Scharenberg, K. (2010). Lernentwicklung in leistungshomogenen und -heterogenen Schulklassen. In W. Bos, E. Klieme \& O. Koller (Hrsg.), Schulische Lerngelegenheiten und Kompetenzentwicklung (S. 173-194). Münster u.a.: Waxmann.

Budde, J. (2012). Die Rede von der Heterogenität in der Schulpädagogik. Diskursanalytische Perspektiven. Forum Qualitative Sozialforschung/Forum: Qualitative Social ResearchOnline, 13 (2), Art. 16. Zugriff am 28.08.2012 unter http://www.qualitative-research.net/ index.php/fqs/article/view/1761/3358 
Diaz-Bone, R. (2006). Zur Methodologisierung der Foucaultschen Diskursanalyse. Historical Social Research, 31 (2), 243-274.

Eckhart, M. (2002). Unterricht in heterogenen Schulklassen. Theoretische Überlegungen und explorative Untersuchung unter besonderer Berücksichtigung von Kindern mit Schulleistungsschwächen. Vierteljahresschrift für Heilpädagogik und ihre Nachbargebiete, $71(2), 152-172$.

Edelmann, D. (2006). Pädagogische Professionalität im transnationalen sozialen Raum. Eine Studie über Sichtweisen und Erfahrungen von Primarlehrpersonen in Bezug auf die kulturelle Heterogenität ihrer Schulklassen. In C. Allemann-Ghionda (Hrsg.), Kompetenzen und Kompetenzentwicklung von Lehrerinnen und Lehrern (Zeitschrift für Pädagogik, Beiheft 51, S. 235-249). Weinheim u.a.: Beltz.

Fachportal Pädagogik (2012). Policy der FIS Bildung Literaturdatenbank. Zugriff am 24.04.2012 unter http://www.fachportal-paedagogik.de/fis_bildung/fis_policy.html

Huber, C. (2009). Soziale Ausgrenzung in der Integration von Schülern mit sonderpädagogischem Förderbedarf. Zusammenhang von Persönlichkeit, Gruppenheterogenität und sozialer Ausgrenzung. Empirische Pädagogik, 23 (2), 170-190.

Hugener, I., Krammer, K. \& Pauli, C. (2008). Kompetenzen der Lehrpersonen im Umgang mit Heterogenität: Differenzierungsmassnahmen im Mathematikunterricht. In M. Gläser-Zikuda \& J. Seifried (Hrsg.), Lehrerexpertise. Analyse und Bedeutung unterrichtlichen Handelns (S. 47-66). Münster u.a.: Waxmann.

Krüger-Potratz, M. (2011). Intersektionalität. In H. Faulstich-Wieland (Hrsg.), Umgang mit Heterogenität und Differenz (S. 183-200). Baltmannsweiler: Schneider Verlag.

Lang, E., Grittner, F., Rehle, C. \& Hartinger, A. (2010). Das Heterogenitätsverständnis von Lehrkräften im jahrgangsgemischten Unterricht der Grundschule. In J. Hagedorn, V. Schurt, C. Steber \& W. Waburg (Hrsg.), Ethnizität, Geschlecht, Familie und Schule (S. 315-331). Wiesbaden: VS Verlag für Sozialwissenschaften.

Lanphen, J. (2011). Kooperatives Lernen und Integrationsforderung. Eine theoriegeleitete Intervention in ethnisch heterogenen Schulklassen. Münster u.a.: Waxmann. Dissertation, Philipps-Universität Marburg.

Leiprecht, R. \& Lutz, H. (2006). Intersektionalität im Klassenzimmer: Ethnizität, Klasse, Geschlecht. In R. Leiprecht \& A. Kerber (Hrsg.), Schule in der Einwanderungsgesellschaft. Ein Handbuch (S. 218-234). Schwalbach: Wochenschau Verlag.

Lindemann, S., Schwanenberg, J. \& Bos, W. (2011). Heterogenität im Lichte rezenter Schulleistungsstudien. In R. Hinz \& R. Walthes (Hrsg.), Verschiedenheit im Diskurs (S. 74-96). Tübingen: Narr Francke Attempto Verlag.

Martschinke, S. \& Kammermeyer, G. (2003). Jedes Kind ist anders. Jede Klasse ist anders. Ergebnisse aus dem KILIA-Projekt zur Heterogenität im Anfangsunterricht. Zeitschrift für Erziehungswissenschaft, 6 (2), 257-275.

Prengel, A. (2007). Im Schwebezustand: Schulen und transgressive Lebenswelten. Ansätze in Forschung und Lehre. Schweizerische Zeitschrift für Bildungswissenschaften, 29 (3), 363-378.

Reinhardt, C. \& Rosen, E. (2004). Einfluss der Altersheterogenität auf Kleingruppenarbeit. In W. Bos (Hrsg.), Heterogenität (S. 245-252). Münster, Westfalen u.a.: Waxmann.

Schenz, C. (2011). Lehrerinnenexpertise im Umgang mit Heterogenität. In C. Schenz, S. Rosebrock \& M. Soff (Hrsg.), Von der Begabtenförderung zur Begabungsgestaltung. Vom kreativen Umgang mit Begabungen in der Mathematik (S. 11-25). Berlin u.a.: LIT.

Schneider, H. (2009). Soziale Heterogenität und Leseleistung. Wenn Schriftaneignung trotzdem gelingt. In H.-U. Grunder \& A. Gut (Hrsg.), Zum Umgang mit Heterogenität in der Schule (Bd. 1, S. 67-81). Baltmannsweiler: Schneider-Verlag Hohengehren.

Schratz, M., Schwarz, J. F. \& Westfall-Greiter, T. (2011). Personale Bildungsprozesse in heterogenen Gruppen. Zeitschrift für Bildungsforschung, 1 (1), 25-39. 
Schründer-Lenzen, A. (2008). Bedingungen guten Unterrichts für heterogene Lerngruppen - Befunde der empirischen Forschung. In H. Kiper, S. Miller, C. Palentien. \& C. Rohlfs (Hrsg.), Lernarrangements für heterogene Gruppen - Lernprozesse professionell gestalten (S. 106-126). Bad Heilbrunn: Klinkhardt.

Schwippert, K., Bos, W. \& Lankes, E.-M. (2004). Heterogenität und Chancengleichheit am Ende der vierten Jahrgangsstufe in den Ländern der Bundesrepublik Deutschland und im internationalen Vergleich. In W. Bos, E.-M. Lankes, M. Prenzel, K. Schwippert, R. Valtin \& G. Walther (Hrsg.), IGLU. Einige Länder der Bundesrepublik Deutschland im nationalen und internationalen Vergleich (S. 165-190). Münster, Westfalen: Waxmann.

Sievers, I. (2009). Individuelle Wahrnehmung, nationale Denkmuster. Einstellungen deutscher und französischer Lehrkräfte zu Heterogenität im Unterricht (1. Auflage). Frankfurt, Main: Brandes \& Apsel. Dissertation, Universität Hannover.

Steinke, M. \& Badura, B. (2011). Präsentismus. Ein Review zum Stand der Forschung. Zugriff am 25.04.2012 unter http://www.gesundheitsfoerderung.ch/pdf_doc_xls/d/betriebliche_ gesundheitsfoerderung/grundlagen_wissen/Praesentismus_2011.pdf

Stöger, H. \& Ziegler, A. (2012). Heterogenität und Inklusion im Unterricht. In L. Haag \& S. Rahm (Hrsg.), Studienbuch Schulpädagogik. Bad Heilbrunn: Klinkhardt/UTB.

Streckeisen, U., Hänzi, D. \& Hungerbühler, A. (2009). Fördern, Selektion und der Umgang mit Heterogenität. Deutungsmuster von Lehrpersonen in soziologischer Hinsicht. In H.-U. Grunder \& A. Gut (Hrsg.), Zum Umgang mit Heterogenität in der Schule (Bd. 1, S. 128-147). Baltmannsweiler: Schneider-Verlag Hohengehren.

Urech, C. (2010). Die heterogene Schulklasse. Fallstudien zum pädagogischen Handeln in Basisstufen. Zürich: Rüegger. Dissertation, Universität Freiburg.

Warwas, J. \& Kunter, M. (2011). Individuelle Förderung in heterogenen Klassen. Im Projekt ,Igel' werden adaptive Lernumgebungen in der Grundschule evaluiert. Forschung Frankfurt, 29 (1), 14-15.

Wenning, N. (2007). Heterogenität als Dilemma für Bildungseinrichtungen. In S. Boller, E. Rosowski \& T. Stroot (Hrsg.), Heterogenität in Schule und Unterricht. Handlungsansätze zum pädagogischen Umgang mit Vielfalt (S. 21-31). Weinheim und Basel: Beltz.

Wirszing, D. \& Weichert, W. (2008). Homogene oder heterogene Schulen oder Klassen? Grundschulmagazin, 76 (3), 11-12.

Wischer, B. (2001). Lernen in heterogenen Gruppen aus Schülersicht. Ausgewählte Ergebnisse aus der Absolventenstudie. In I. Demmer-Dieckmann \& B. Struck (Hrsg.), Gemeinsamkeit und Vielfalt. Pädagogik und Didaktik einer Schule ohne Aussonderung (S. 227-246). Weinheim; München: Juventa.

Wischer, B. (2007). Wie sollen LehrerInnen mit Heterogenität umgehen? Über «programmatische Fallen» im aktuellen Reformdiskurs. Die deutsche Schule, 99 (4), 422-433.

Wischer, B. (2009). Der Diskurs um Heterogenität und Differenzierung. Beobachtungen zu einem schulpädagogischen «Dauerbrenner». In B. Wischer \& K.-J. Tillmann (Hrsg.), Erziehungswissenschaft auf dem Prüfstand. Schulbezogene Forschung und Theoriebildung von 1970 bis heute (S. 69-93). Weinheim, München: Juventa.

Schlagworte: Heterogenität, Heterogenitätskategorien, empirische Studien 


\section{La notion d'hétérogénéité dans des études empiriques}

Résumé

Lorsque le titre d'une étude empirique comprend la notion d'hétérogénéité, quels objets et quelles catégories d'hétérogénéité sont examinés? La recherche rapportée dans cet article contribue à répondre à cette question en s'appuyant sur une revue systématique et sur l'analyse de contenu d'un corpus d'études empiriques. À partir d'un ensemble de critères, 21 travaux ont été catégorisés dans l'une des trois thématiques génériques suivantes: 1. L'hétérogénéité d'une population d'élèves selon différentes catégories d'hétérogénéité et niveaux d'analyse. Il ressort que la catégorie d'hétérogénéité la plus fréquemment étudiée concerne celle liée à la performance des élèves. 2. L'implémentation, l'évaluation et le développement de méthodes d'enseignement intégratives. 3. Les théories subjectives des enseignants à propos de l'hétérogénéité des élèves.

Mots-clés: Hétérogénéité, catégories d'hétérogénéité, revue d'études empiriques, analyse de contenu

\section{II concetto di eterogeneità in studi empirici}

\section{Riassunto}

Su quali argomenti e quali categorie di eterogeneità si concentrano le ricerche quando nel titolo di uno studio empirico viene utilizzato il termine «eterogeneità»? Questa domanda viene approfondita sulla base di una ricerca sistematica su banche dati e un'analisi dei contenuti di studi di ricerca. 21 studi, che possono essere suddivisi nei tre punti tematici cruciali di seguito riportati, hanno mostrato una corrispondenza con i parametri definiti. 1. L'eterogeneità degli studenti rispetto a differenti categorie di eterogeneità e livelli di analisi. In tale contesto, l'eterogeneità del rendimento è la categoria esaminata più spesso. 2. L'applicazione, la valutazione e l'evoluzione di metodi d'insegnamento integrativi. 3. Le teorie soggettive dei docenti rispetto all'eterogeneità dei propri studenti.

Parole chiave: Eterogeneità, categorie di eterogeneità, studi empirici 


\section{The Notion of «Heterogeneity" in Empirical Studies}

\section{Summary}

When the term "heterogeneity» occurs in the title of an empirical study, wich topics and wich heterogeneity categories are being studied? This question is explored by means of a systematic database research and content analysis of empirical studies. With respect to defined criteria, 21 studies are classified into the following three key topics: 1 . Student heterogeneity with respect to different heterogeneity categories and levels of analysis. It appears that performance-related heterogeneity is the most frequently investigated category. 2 . The implementation, evaluation and development of inclusive teaching methods. 3. Teachers' subjective theories about students' heterogeneity.

Keywords: Heterogeneity, heterogeneity categories, empirical studies review 